\title{
Idle Speculation or Proficient Prognosis? How to Employ Celebrity Endorsement Models Smartly: An Abstract
}

\author{
Klaus-Peter Wiedmann and Walter von Mettenheim
}

\begin{abstract}
Celebrity endorsement is a promising form of marketing communication. Verily, a celebrity endorsement can yield 30 times its costs. On the other hand, a failed celebrity endorsement can likewise cause financial damage: In 1989, PepsiCo had to withdraw its endorsement of pop diva Madonna due to substantial protest and calls for boycott. The loss has been estimated at six million dollars. This ambiguity hints at the fact that celebrity endorsement has to be carried out in a methodical and smart way: In order to help advertising managers to make the right decisions, countless celebrity endorsement models and indices have been designed: Among them are the wellknown, established ones, which are the Source Credibility Model, the Source Attractiveness Model, the Match-Up Hypothesis, the Meaning-Transfer Model, the Q Score, and the Davie-Brown Index. However, there are also lesser known models and indices, which are as follows: the Four Fs, FRED, TEARS, Celebrity Endorsement Model, Dual Entertainment Pass Model, E-Score, and Celebrity Performance Index. All have their virtues and advantages; but which of them are most advisable and reliable? Which cover the critical decision factors of celebrity endorsement in the best way? Additionally the question arises, whether the primacy of the established models is really justifiable. In other words, are the established models and indices really decidedly better than all the rest? Answering these questions is the aim of the study at hand. To this purpose, at first the overlying perspective will be elucidated. Subsequently, a literature review is carried out, in order to reveal all aspects that are relevant for smart celebrity endorsement. Thereby, a list of attributes arises. These constitute the basis for the ensuing assessment of the models and indices. The results imply that concerning models, a combination of Celebrity Endorsement Model and FRED is the best solution for advertising managers. In terms of indices, the Davie-Brown Index and E-Score are best suited. Finally, the results are employed to design implications for advertising managers and point out research gaps.
\end{abstract}

\section{References Available Upon Request}

K.-P. Wiedmann •W. von Mettenheim $(\bowtie)$

Leibniz University of Hannover, Hannover, Germany

e-mail: wiedmann@m2.uni-hannover.de; vonmettenheim@m2.uni-hannover.de

(C) Academy of Marketing Science 2018 